\title{
Functional localization of sensorimotor cortex by somatosensory evoked potentials produced by femoral nerve stimulation
}

\author{
Akifumi Suzuki, M.D., D.M.Sc., Kimio Yoshioka, C.E., Hiromi Nishimura, B.E., C.E., and \\ Nobuyuki Yasui, M.D., D.M.Sc.
}

Department of Surgical Neurology, Research Institute for Brain and Blood Vessels, Akita, Japan

Cortical somatosensory evoked potentials (SSEPs) can be used to localize the central sulcus during a craniotomy. In particular, contralateral median nerve stimulation producing SSEPs can disclose the location of the central sulcus around the sensorimotor hand representation area. However, the median nerve cannot be stimulated in patients who undergo craniotomy at locations other than the hand representation area. The present study attempts to localize the central sulcus in the lateral surface of the brain near the interhemispheric fissure by stimulating the contralateral femoral nerve to produce SSEPs. Somatosensory evoked potentials were recorded between the superior lip of the interhemispheric fissure and 1.5 to $2 \mathrm{~cm}$ laterally in the cortex. Only seven of the 12 patients studied showed a phase reversal of the initial component across the central sulcus. The polarity was negative in the postcentral gyrus and positive in the precentral gyrus. The other five patients did not show a phase reversal of the initial component across the central sulcus. The amplitude was highest in the postcentral gyrus and the polarity was positive. Based on these results, the authors hypothesize that stimulating the contralateral femoral nerve to produce SSEPs and then analyzing the distribution of the SSEPs may provide a method for functional localization of the sensorimotor cortex around the interhemispheric fissure during craniotomy.

\section{Key Words * somatosensory evoked potentials * functional localization * sulcus * sensorimotor cortex * femoral nerve}

Stimulation of the median nerve to produce somatosensory evoked potentials (SSEPs) has been used during craniotomy to functionally localize the sensorimotor cortex.[2-4,6,13,14] Cortical SSEPs produced by stimulation of the median nerve can localize the sensorimotor hand representation area located away from the interhemispheric fissure. When craniotomy is performed around the central sulcus near the interhemispheric fissure, stimulation of peripheral nerves in the lower extremity, such as the tibial and peroneal nerves, to produce SSEPs is preferred for localization of the sensorimotor cortex. However, the source that generates the cortical component evoked by stimulation of the tibial and peroneal nerves is situated in the medial surface of the cerebral cortex within the interhemispheric fissure.[1,5,7,10,11] Therefore, stimulation of these nerves does not serve to localize the sensorimotor cortex in the lateral surface of the cerebral cortex near the interhemispheric fissure.

Wang, et al.,[12] recorded scalp SSEPs by stimulating the femoral nerve and suggested that the cortical component might originate from the superior lip of the interhemispheric fissure just over the lateral 
cortex. In the present study, cortical SSEPs produced by stimulation of the femoral nerve were recorded. The possibility of using these SSEPs to localize the sensorimotor cortex in the lateral cortex near the interhemispheric fissure is discussed.

\section{CLINICAL MATERIAL AND METHODS}

\section{Patient Population}

The patient population consisted of three males and nine females aged between 17 and 63 years (average $45 \pm 17$ years). The patients' mean height was $156.2 \pm 7.3 \mathrm{~cm}$. Eleven of the 12 patients had brain tumors and the other patient had an arteriovenous malformation (Table 1). Cortical SSEPs were produced by stimulating the femoral nerve in all 12 patients who underwent craniotomy, which exposed the interhemispheric fissure.

\section{TABLE1}

CLINICAL DIAGNOSES AND FIND NGS IN THE INT ML RESULTS OF CORTICAL SOMATOSENSORV EVOKED POTENTIALS*

\begin{tabular}{|c|c|c|c|c|c|c|c|c|c|c|}
\hline \multirow[b]{2}{*}{ Case } & \multirow[b]{2}{*}{$\begin{array}{c}\text { Age (ys), } \\
\text { Sex }\end{array}$} & \multirow[b]{2}{*}{ Diagnosis } & \multicolumn{4}{|c|}{ Femoral Nerve Stim ulation } & \multicolumn{4}{|c|}{ Medan Nerve Stim dation } \\
\hline & & & $\begin{array}{l}\text { Latency } \\
\text { (msec) }\end{array}$ & $\begin{array}{c}\text { Amplitude } \\
\left(\mu, v^{\prime}\right)\end{array}$ & Pol. & Rev. & $\begin{array}{l}\text { Latency } \\
\text { (msec) }\end{array}$ & $\begin{array}{c}\text { Amplitude } \\
\left(\mu V^{\prime}\right)\end{array}$ & Pol. & Rev \\
\hline 1 & $63, M$ & It frontal glioma & 22.5 & 8.4 & N & + & 23.4 & 15.4 & N & + \\
\hline 2 & $61, F$ & It frontal glioma & 24.8 & 3.2 & N & + & 23.0 & 4.1 & N & + \\
\hline 3 & $26, F$ & rt frontal glioma & 22.2 & 3.4 & N & + & 20.5 & 17.2 & N & + \\
\hline 4 & $17, \mathrm{~F}$ & rt frontal glioma & 21.1 & 1.3 & $N$ & + & 19.4 & 17.8 & $\mathrm{~N}$ & + \\
\hline 5 & $34, F$ & rt thalamic glioma & 22.6 & 3.1 & N & + & 21.1 & 9.1 & N & + \\
\hline 6 & $62, F$ & rt frontal meningioma & 24.6 & 4.3 & N & + & 21.6 & 3.2 & N & + \\
\hline 7 & $35, F$ & It parietal AUM & 20.4 & 2.3 & $N$ & + & 19.0 & 15.0 & N & + \\
\hline 8 & $22, F$ & rt frontal glioma & 23.0 & 6.8 & $\mathrm{P}$ & - & 21.0 & 6.0 & N & + \\
\hline 9 & $57, \mathrm{~F}$ & rt frontal glioma & 22.9 & 11.0 & $P$ & - & 21.0 & 24.0 & N & + \\
\hline 10 & $63, M$ & rt parie tal glioma & 25.2 & 7.1 & $\mathrm{P}$ & - & 22.0 & 30.8 & N & + \\
\hline 11 & $44, M$ & It lat. ve meningioma & 24.8 & 14.4 & $\mathrm{P}$ & - & 22.5 & 27.0 & N & + \\
\hline 12 & $61, \mathrm{~F}$ & It frontal meningiom a & 21.8 & 22.4 & $\mathrm{P}$ & - & 20.2 & 7.5 & $N$ & + \\
\hline
\end{tabular}

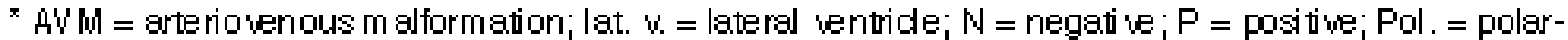
it $y$, Rev. = phase reversal.

\section{Surgical Protocol}

A general anesthetic consisting of $60 \%$ nitrous oxide in oxygen and $0.5 \%$ to $1.0 \%$ enflurane was administered and a wide area of the lateral surface of the brain between the sylvian fissure and interhemispheric fissure was exposed via craniotomy and reflection of the dura. Needle electrodes were inserted into the skin over the inguinal ligament $1.5 \mathrm{~cm}$ lateral to the femoral artery to stimulate the femoral nerve contralateral to the craniotomy. The cathode was placed $2 \mathrm{~cm}$ proximal to the anode. Electrical stimuli consisting of 0.2 -msec constant-current pulses were delivered. The stimulus intensity was adjusted to produce a small twitch in quadriceps femoris at a stimulus rate of $5 \mathrm{~Hz}$. A silicone sheet holding four recording electrodes (10 mm interelectrode distance) was placed on the cortical surface. An average of 250 responses was made using a frequency filter bandpass of 5 to $3000 \mathrm{~Hz}$. The analyzing time was $40 \mathrm{msec}$ after the stimulus. Cortical SSEPs were recorded twice so as to assess their reproducibility. A reference electrode was placed in the temporalis muscle adjacent to the craniotomy margin. The initial deflection of the cortical SSEPs was designated as a N1 or P1 wave, depending on the polarity $(\mathrm{N}=$ negative and $\mathrm{P}=$ positive $)$.

First, the central sulcus was localized using the SSEPs recorded from median nerve stimulation. The 
methods used to record and determine the location of the central sulcus using median nerve stimulation are described in a previous report.[8] After localizing the central sulcus in the lateral surface of the brain located away from the interhemispheric fissure, SSEPs produced by femoral nerve stimulation were recorded. Next, cortical stimulation $(10-15 \mathrm{~mA}$, at a $0.2-\mathrm{msec}$ duration with $50-\mathrm{Hz}$ trains applied bipolarly) was performed to identify the motor cortex.

\section{RESULTS}

In all patients, SSEPs produced by femoral nerve stimulation could be recorded. Amplitudes of the potentials were highest in the postcentral gyrus at the superior lip of the interhemispheric fissure just over the lateral cortex. The mean peak latency and amplitude of the initial component were measured from the recording at this point (Table 1). Femoral nerve SSEPs could be recorded between the superior lip of the interhemispheric fissure and 1.5 to $2 \mathrm{~cm}$ laterally in the cortex. Phase reversal of the initial deflection of cortical femoral nerve SSEPs across the central sulcus was observed in only seven of the 12 cases.

In the seven patients showing phase reversal across the central sulcus, the polarity of the postcentral gyrus was negative and that of the precentral gyrus was positive. This same trend was noted for the median nerve SSEPs. The mean peak latency of the N1 wave of femoral nerve SSEPs was $22.6 \pm 1.5$ msec and the mean peak amplitude was $3.7 \pm 2.1 \mu \mathrm{V}$ in the postcentral gyrus. The mean peak latency of the N1 wave of the median nerve SSEPs was $21.1 \pm 1.5 \mathrm{msec}$ and the mean peak amplitude was $11.7 \pm$ $5.7 \mu \mathrm{V}$. The mean height of these seven patients was $154.5 \pm 5.2 \mathrm{~cm}$.

In the other five patients who showed no phase reversal across the central sulcus, the polarity of the initial deflection was positive. Furthermore, the amplitude of the P1 wave was highest in the postcentral gyrus. The mean peak latency of the P1 wave was $23.5 \pm 1.3 \mathrm{msec}$ and the mean peak amplitude was $12.3 \pm 5.8 \mu \mathrm{V}$ in the postcentral gyrus. The mean peak latency of the N1 wave of median nerve SSEPs was $21.3 \pm 0.8 \mathrm{msec}$ and the mean peak amplitude was $19.1 \pm 10.3 \mu \mathrm{V}$. The mean height of these 5 patients was $158.6 \pm 9 \mathrm{~cm}$.

The peak latency of the P1 wave of the femoral nerve SSEPs in patients without phase reversal was slightly longer than that of the N1 wave of the femoral nerve SSEPs in patients with phase reversal. However, the correlative analysis between the two groups revealed no statistically significant differences $(\mathrm{t}=1.03$, p greater than 0.2$)$. The peak latency of the N1 wave of the median nerve SSEPs in patients without phase reversal of femoral nerve SSEPs was not significantly different from that in patients with phase reversal of femoral nerve SSEPs $(t=0.24$, $p$ greater than 0.5$)$. The peak amplitude of the P1 wave of the femoral nerve SSEPs in patients without phase reversal was significantly higher than that of the $\mathrm{N} 1$ wave of the femoral nerve SSEPs in patients with phase reversal $(\mathrm{t}=2.87$, $\mathrm{p}$ less than 0.05$)$. Furthermore, the peak amplitude of the N1 wave of the median nerve SSEPs in patients without phase reversal was higher than that in patients with phase reversal. However, this difference was not significant $(\mathrm{t}=1.45$, $\mathrm{p}$ greater than 0.1$)$. The average height of patients showing femoral nerve SSEPs without phase reversal was not significantly different from that of patients showing femoral nerve SSEPs with phase reversal $(\mathrm{t}=0.91$, $\mathrm{p}$ greater than 0.2$)$.

\section{ILLUSTRATIVE CASES}

\section{Case 3}

This 26-year-old woman experienced generalized convulsions on January 23, 1994. She was admitted to our institution on February 1, 1994. 
Examination. Neurological examination revealed no apparent deficit. Magnetic resonance imaging showed a well-defined lesion in the right frontal lobe (Fig. 1). Cerebral angiography revealed a vascular mass lesion with an arteriovenous shunt in the right frontal lobe. Based on these examinations, the patient was diagnosed as having a high-grade astrocytoma in the right frontal lobe.
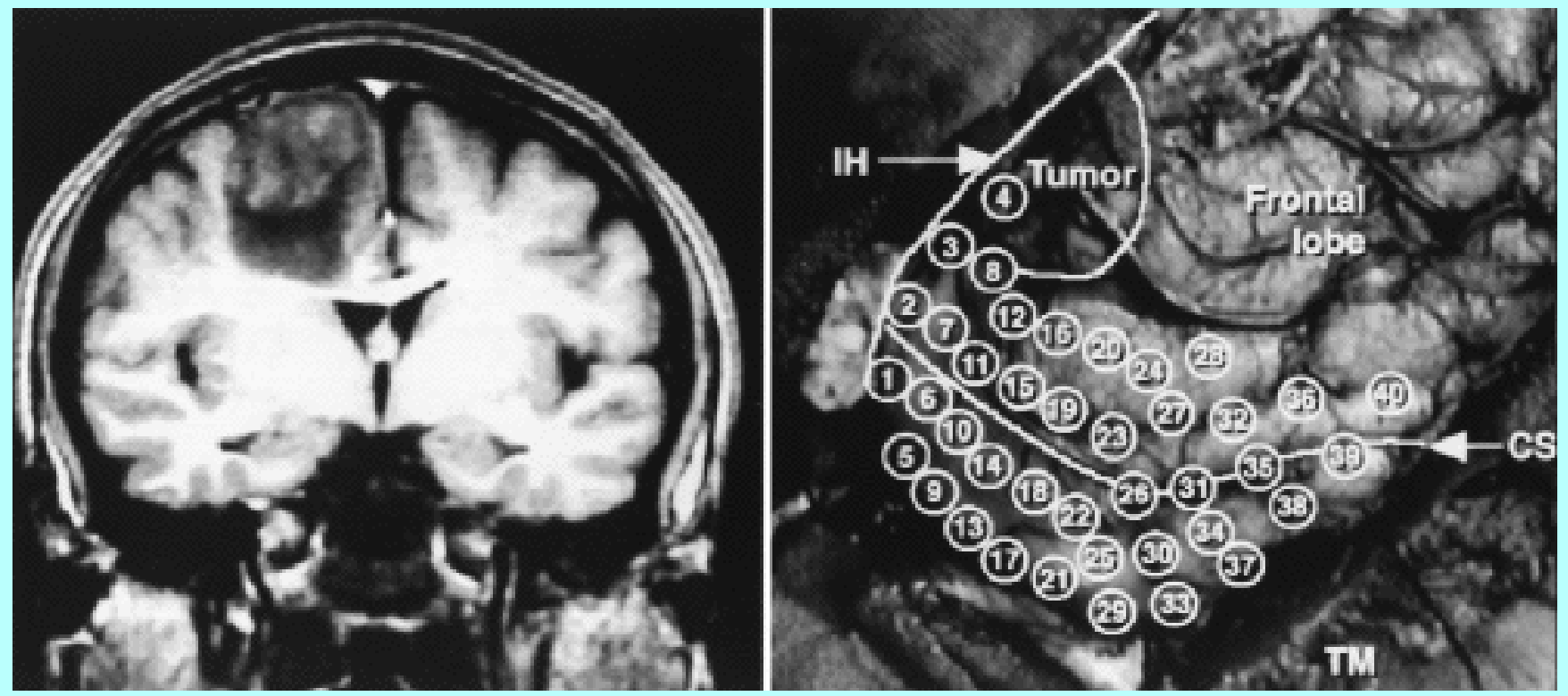

Fig. 1. Case 3. Left: Gadolinium-enhanced magnetic resonance image showing a mass in the right frontal lobe. Right: Intraoperative photograph of the brain surface showing placement of electrodes for recording somatosensory evoked potentials. Cortical stimulation at Electrode 2 elicited electromyographic (EMG) responses in the left quadriceps femoris muscles and stimulation at Electrode 23 elicited EMG responses in the left thumb. $\mathrm{CS}=$ central sulcus; $\mathrm{IH}=$ interhemispheric fissure; $\mathrm{TM}=$ temporalis muscle.

Operation. On February 9, the patient underwent a right frontoparietal craniotomy. After incision of the dura, cortical SSEPs produced by electrical stimulation of left median nerve and left femoral nerve were recorded. Figure 1 shows the electrode placement for recording SSEPs. Recordings of SSEPs during left median nerve stimulation produced isolatency lines for $\mathrm{N} 1$ waves that peaked at $20.5 \mathrm{msec}$ and left femoral nerve stimulation produced isolatency lines that peaked at $22.2 \mathrm{msec}$. Phase reversal of the initial deflections in both median nerve SSEPs and femoral nerve SSEPs were seen in this patient. Figure 2 shows the distribution of potentials in the initial deflections. The patient's tumor was situated near the interhemispheric fissure. The initial distribution of median nerve SSEPs revealed that the phase reversal occurred away from the brain tumor. The distribution of the femoral nerve SSEPs further revealed that the phase reversal occurred near the interhemispheric fissure. Furthermore, cortical stimulation at the location of Electrode 2 elicited electromyographic (EMG) responses in the left quadriceps femoris muscles and stimulation at the location of Electrode 23 elicited EMG responses in the left thumb. Based on these results, the sensorimotor cortex could be localized and the tumor was resected without injury to the motor cortex. 

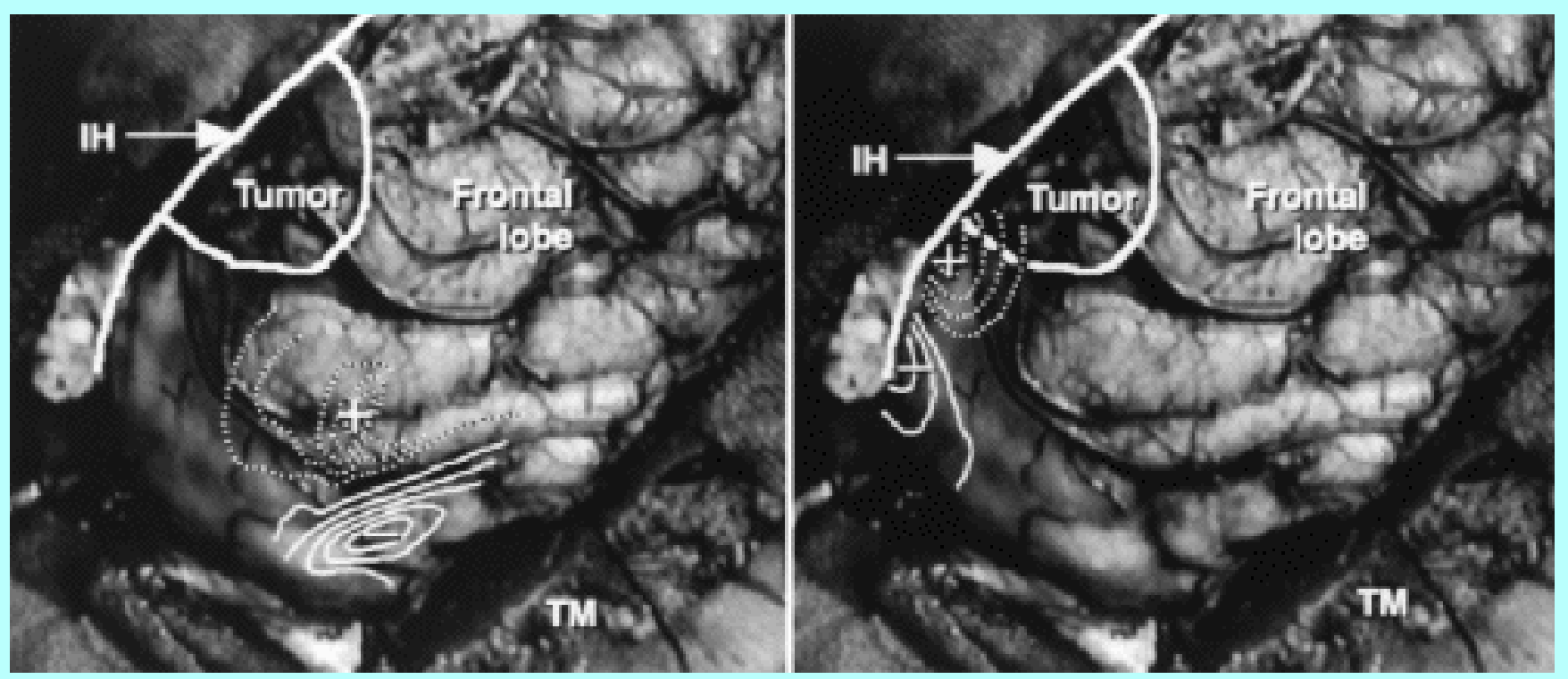

Fig. 2. Case 3. Intraoperative photographs showing the amplitude of the N1 waves of cortical somatosensory evoked potentials. The continuous line indicates the isopotential of negative polarity and dotted line indicates that of positive polarity. Left: Left median nerve stimulation ( $3 \mathrm{mV} / \mathrm{step})$. Right: Left femoral nerve stimulation $(1 \mathrm{mV} / \mathrm{step}) . \mathrm{IH}=$ interhemispheric fissure; $\mathrm{TM}=$ temporalis muscle.

Postoperative Course and Pathological Findings. The pathological diagnosis of the tumor was that of a Grade 3 astrocytoma. The patient was discharged without any neurological deficit on February 24.

\section{Case 11}

This 44-year-old man reported headaches after he suffered head trauma on June 22, 1989. Computerized tomography analysis was performed on July 13 and revealed a left lateral ventricular tumor (Fig. 3). He was admitted on that day to our institution.
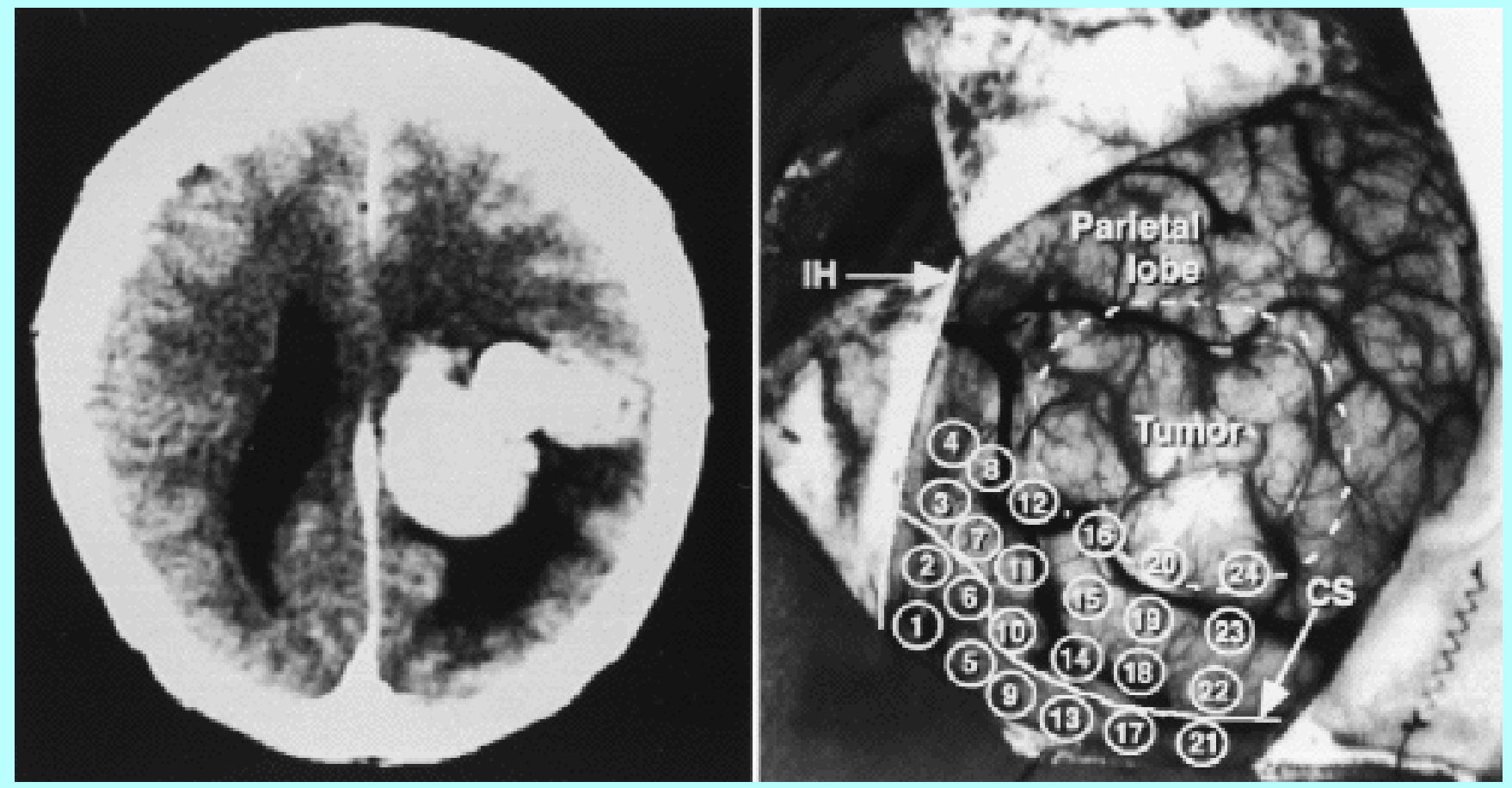

Fig. 3. Case 11. Left: Contrast-enhanced computerized tomography scan revealing a mass in the left lateral ventricle and parietal lobe. Right: Intraoperative photograph of the brain 
surface showing placement of electrodes for recording somatosensory evoked potentials. Cortical stimulation at Electrode 2 elicited electromyographic (EMG) responses in the right quadriceps femoris muscles and stimulation at Electrode 9 elicited EMG responses in the right thumb. $\mathrm{IH}=$ interhemispheric fissure; $\mathrm{CS}=$ central sulcus.

Examination. Further neurological examination revealed a right homonymous hemianopsia, dyscalculia, and dyslexia. Left cerebral angiography demonstrated a vascular mass lesion supplied by the anterior choroidal artery. Based on these examinations, the patient was diagnosed with a meningioma of the left lateral ventricle.
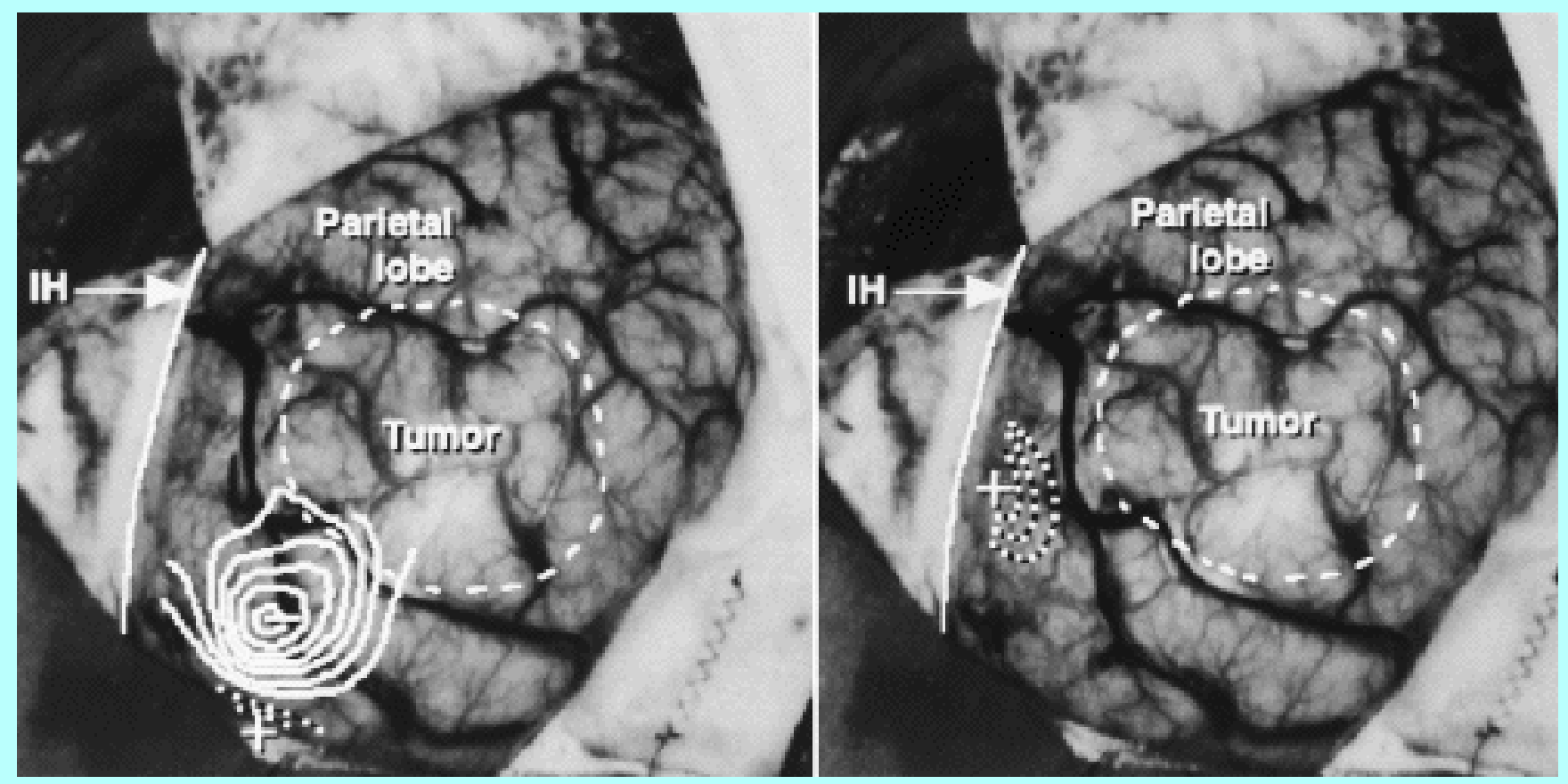

Fig. 4. Case 11. Intraoperative photographs showing the amplitudes of the initial components of cortical somatosensory evoked potentials. The continuous line indicates the isopotential of negative polarity and dotted line indicates that of positive polarity. Left: Right median nerve stimulation ( $4 \mu \mathrm{V} / \mathrm{step})$. Right: Right femoral nerve stimulation (6 $\mu \mathrm{V} / \mathrm{step}) . \mathrm{IH}=$ interhemispheric fissure.

Operation. On July 24, the patient underwent a left frontoparietal craniotomy. After incision of the dura, cortical SSEPs produced by electrical stimulation of the right median nerve and right femoral nerve were recorded. Figure 4 shows the placement of electrodes for recording SSEPs. Recordings of SSEPs during right median nerve stimulation produced isolatency lines for N1 waves that peaked at $22.5 \mathrm{msec}$ and right femoral nerve stimulation produced isolatency lines for P1 waves that peaked at $24.8 \mathrm{msec}$. The patient showed phase reversal of the initial deflections in median nerve SSEPs, but not in femoral nerve SSEPs. The initial distribution of median nerve SSEPs revealed the location of the central sulcus based on the phase reversal. In contrast, femoral nerve SSEP phase reversal did not reveal the location of the central sulcus. The initial femoral nerve SSEP was positive (P1). The motor cortex was localized by electrical stimulation of the cortex. Cortical stimulation at the location of Electrode 2 elicited EMG responses in the right quadriceps femoris muscles and stimulation at the location of Electrode 9 elicited EMG responses in the right thumb. The amplitude of the P1 wave of the femoral nerve SSEPs was highest at Electrode 3. Based on this finding, the sensory cortex was localized. After localization of the sensorimotor cortex, total tumor resection was accomplished using a transcortical approach via the left parietal lobe. 
Postoperative Course and Pathological Findings. The pathological diagnosis of the tumor was that of a meningioma. The patient was discharged without additional neurological deficit on August 17.

\section{DISCUSSION}

Somatosensory evoked potentials produced by median nerve stimulation have been used for functional localization of the sensorimotor cortex during surgery. $[3,4,6,13,14]$ However, the validity of this procedure is restricted to the hand representation area. We previously reported a patient with a brain tumor in whom median nerve SSEPs showed phase reversal across the precentral sulcus at a location away from the hand representation area; [8] thus, we conclude that stimulation of another peripheral nerve is necessary for functional localization of the sensorimotor cortex during surgery near the interhemispheric fissure. Somatosensory evoked potentials elicited by tibial or peroneal nerve stimulation are preferred for localizing the sensorimotor cortex within the interhemispheric fissure.[1,5,7,10,11] However, for surgery at a location just lateral to the interhemispheric fissure, stimulation of the tibial or peroneal nerve cannot be used for functional localization of the sensorimotor cortex. Wang, et al.,[12] reported that scalp SSEPs could be produced by femoral nerve stimulation and the source that generated the cortical potential was believed to be centrally located at the superior lip of the fissure just over the lateral cortex. Subsequently, SSEPs produced by femoral nerve stimulation have been recorded in the lateral cortex during surgery, thus enabling the study of functional localization of the sensorimotor cortex. Our hypothesis is that the functional localization of the sensorimotor cortex is possible at a lateral location near the interhemispheric fissure by recording SSEPs produced by femoral nerve stimulation.

The present results revealed two patterns of initial deflection of cortical femoral nerve SSEPs. Initial deflection of femoral nerve SSEPs with phase reversal across the central sulcus was negative in the postcentral gyrus and positive in the precentral gyrus. This pattern is the same as the initial deflection of median nerve SSEPs in and around the hand representation area. The phase reversal of initial deflection of median nerve SSEPs is believed to result from a horizontal dipole produced in the posterior bank (Area $3 b$ ) of the central sulcus.[6,13] A study of cerebral magnetic responses to median nerve stimulation supports this belief.[9] Similarly, the initial deflection of femoral nerve SSEPs with phase reversal is also considered to be generated by a horizontal dipole produced in Area 3b. Wang, et al.,[12] reported the first localized postrolandic component at the P2 wave (P26). Their Group I patients showed a phase reversal counterpart (N26) in the frontal area.[12] They believe that this component (P26/N26) is generated by a horizontal dipole in Area $3 b$ or by multiple dipoles. However, the polarity of the P26/N26 wave in their study was opposite to the initial deflection of femoral nerve SSEPs in the present study. Recordings of femoral nerve SSEPs from a normal volunteer in Group I (Fig. 3 in Wang, et al.) showed phase reversal at the N1 wave (N19), similar to the negative polarity in the parietal area and positive polarity in the frontal area.[12] The initial deflection of femoral nerve SSEPs with phase reversal seen in the present study might coincide with the N1 wave (N19) of the femoral nerve SSEPs in Group I studied by Wang, et al.

Five patients in the present study did not show phase reversal across the central sulcus and the polarity of the initial deflection was positive. The amplitude was highest at the postcentral gyrus. By analyzing the distribution of the amplitude of the positive deflection, the central sulcus was localized. However, in this case, generation of the initial deflection of this pattern cannot be explained by a horizontal dipole in Area $3 b$. The positive deflection is likely generated primarily at the surface of the postcentral gyrus (Areas 1 and 2) and might coincide with the P2 wave (P26) in Group II studied by Wang, et al.[12] In the present study, the amplitude of the P1 wave of the femoral nerve SSEPs in patients without phase reversal was 
significantly higher than that of the N1 wave of the femoral nerve SSEPs in patients with phase reversal. This result seems to support the belief that the P1 wave of the femoral nerve SSEPs in patients without phase reversal is the potential which is generated perpendicularly in the lateral surface of the postcentral gyrus. Thus, variations may exist in the recipient area of thalamocortical projections in the femoral representation area.

Femoral nerve SSEP recordings were primarily distributed in the restricted area of the lateral cortex near the interhemispheric fissure. However, by analyzing the distribution of femoral nerve SSEPs, the sensorimotor cortex was functionally localized. Thus, femoral nerve SSEPs can be used to localize the sensorimotor cortex for craniotomy around the interhemispheric fissure.

\section{Acknowledgment}

The authors wish to thank Miss T. Aburaya for her assistance in preparing this manuscript.

\section{References}

1. Cruse R, Klem G, Lesser RP, et al: Paradoxical lateralization of cortical potentials evoked by stimulation of posterior tibial nerve. Arch Neurol 39:222-225, 1982

2. Dinner DS, Lueders H, Lesser RP, et al: Cortical generators of somatosensory evoked potentials to median nerve stimulation. Neurology 37:1141-1145, 1987

3. Gregorie EM, Goldring S: Localization of function in the excision of lesions from the sensorimotor region. J Neurosurg 61:1047-1054, 1984

4. King RB, Schell GR: Cortical localization and monitoring during cerebral operations. J Neurosurg 67:210-219, 1987

5. Lesser RP, Lueders H, Dinner DS, et al: The source of 'paradoxical lateralization' of cortical evoked potentials to posterior tibial nerve stimulation. Neurology 37:82-88, 1987

6. Lueders H, Lesser RP, Hahn J, et al: Cortical somatosensory evoked potentials in response to hand stimulation. J Neurosurg 58:885-894, 1983

7. Seyal M, Emerson RG, Pedley TA: Spinal and early scalp-recorded components of the somatosensory evoked potential following stimulation of the posterior tibial nerve. Electroenceph Clin Neurophysiol $\mathbf{5 5 : 3 2 0 - 3 3 0 , 1 9 8 3 ~}$

8. Suzuki A, Yasui N: Intraoperative localization of the central sulcus by cortical somatosensory evoked potentials in brain tumor. Case report. J Neurosurg 76:867-870, 1992

9. Tiihonen J, Hari R, Hamalainen M: Early deflections of cerebral magnetic responses to median nerve stimulation. Electroenceph Clin Neurophysiol 74:290-296, 1989

10. Tsumoto T, Hirose N, Nonaka S, et al: Analysis of somatosensory evoked potentials to lateral popliteal stimulation in man. Electroenceph Clin Neurophysiol 33:379-388, 1972

11. Vas GA, Cracco JB, Cracco RQ: Scalp-recorded short latency cortical and subcortical somatosensory evoked potentials to peroneal nerve stimulation. Electroenceph Clin Neurophysiol 52:1-8, 1981 
12. Wang J, Cohen LG, Hallett M: Scalp topography of somatosensory evoked potentials following electrical stimulation of femoral nerve. Electroenceph Clin Neurophysiol 74:112-123, 1989

13. Wood CC, Spencer DD, Allison T, et al: Localization of human sensorimotor cortex during surgery by cortical surface recording of somatosensory evoked potentials. J Neurosurg 68:99-111, 1988

14. Woolsey CN, Erickson TC, Gilson WE: Localization in somatic sensory and motor areas of human cerebral cortex as determined by direct recording of evoked potentials and electrical stimulation. $\mathbf{J}$ Neurosurg 51:476-506, 1979

Manuscript received July 9, 1996.

Accepted in final form August 21, 1996.

Address reprint requests to: Akifumi Suzuki, M.D., D.M.Sc., Department of Surgical Neurology, Research Institute for Brain and Blood Vessels, 6-10, Senshu-kubota-machi, Akita 010 Japan.

Click here to go to Editor's Perspective on 3. 